\title{
Solitary True Vocal Cord Neurofibroma: An Unexpected Pathology
}

\author{
Bidaye $\mathbf{R}^{1}$, Mahmood A ${ }^{1 *}$, Karamchandani $\mathrm{D}^{2}$ and Mriganka $\mathrm{De}^{1}$ \\ ${ }^{1}$ ENT Department, University Hospitals Birmingham, United Kingdom \\ ${ }^{2}$ ENT Department, University Hospital Coventry \& Warwickshire, United Kingdom
}

*Corresponding author: Mr. Ashraf Mahmood, ENT Department, Heartlands Hospital, University Hospital Birmingham NHS Foundation Trust, United Kingdom, Email: ashrafmahmood@yahoo.com

\section{Case Report \\ Volume 4 Issue 1}

Received Date: January 31, 2020

Published Date: February 13, 2020

DOI: $10.23880 /$ mjccs- 16000246

\section{Abstract}

Laryngeal neurofibroma is a rare pathology, usually presents in patients with neurofibromatosis and the most common location being the supraglottis followed by the subglottic area. Our case presented with dysphonia and was found to have a unilateral vocal fold cystic lesion. Histopathological examination after surgical excision diagnosed the benign looking cyst as a neurofibroma of the vocal fold. This extremely rare pathology is only the third case reported in the published literature. Due to the rarity of this diagnosis and the limited clinical information about vocal fold neurofibroma in patients without neurofibromatosis, we are suggesting the possible management in similar cases and the importance of regular follow up taking in consideration the recurrence possibility.

Keywords: Neurofibroma; Vocal Cord; Polyp

\section{Introduction}

Laryngeal neurofibroma is an extremely rare pathology that makes up only $0.03-0.1 \%$ of the benign tumors of the larynx [1]. Of these reported lesions, most are in the supraglottis followed by the subglottic area. Only two cases of isolated neurofibroma of the vocal fold in a patient without neurofibromatosis has been reported in the published literature previously $[2,3]$. We are reporting the third case of this extremely rare pathology.

\section{Case Presentation}

A 65-year-old lady was referred to our Head and Neck clinic for a lesion on the right tonsil which was noticed 3 months back. Upon further questioning the patient also gave a history of voice hoarseness and sore throat for 2 months. She denied any swallowing difficulty, dyspnea or weight loss. She did not have any previous intubation or history of laryngeal trauma. Her medical history was significant for hypertension and hypercholesterolemia and she used to smoke for the last 25 years.
Clinical examination of the throat showed a haemorrhagic cyst on the right tonsil and Fibre-optic Nasal Endoscope (FNE) revealed a left vocal fold lesion. Closer inspection showed that the lesion is arising from the anterior two thirds of the left vocal fold with a wide base attached to the true vocal fold. The movement of the vocal folds was equal bilaterally. There was partial obstruction of the airway caused by the lesion, but the patient had no complaints suggestive of any respiratory compromise. It was provisionally diagnosed as a benign looking left vocal fold cystic lesion and a microlaryngoscopy with excision of the lesion was planned. An excision of the cystic lesion over the right tonsil was also proposed during the same procedure.

Pre-operative haematological investigations including kidney function, electrolytes and full blood count were normal apart from slightly raised white blood cells count of $9.26 \times 10^{9} / \mathrm{L}$ (upper limit of normal $8.40 \times 10^{9} / \mathrm{L}$ ) and elevated neutrophils of $5.43 \times 10^{9} / \mathrm{L}$ (upper limit of normal $4.60 \times 10^{9} / \mathrm{L}$ ). A CT scan was not performed considering the benign appearance of the lesion and the normal mobility of 


\section{Medical Journal of Clinical Trials \& Case Studies}

the vocal cords.

Suspension microlaryngoscopy was performed with a Dedo scope and the lesion was palpated for its consistency, boundaries and depth (Figure 1). A superior cordotomy incision was performed and a mucosal flap was raised. After careful dissection the submucosal lesion was completely excised. The right tonsillar cystic lesion was also excised. Both lesions were sent for histopathological examination. With no immediate post-operative complications, patient was discharged home on the same day with voice rest instructions for 72 hours.

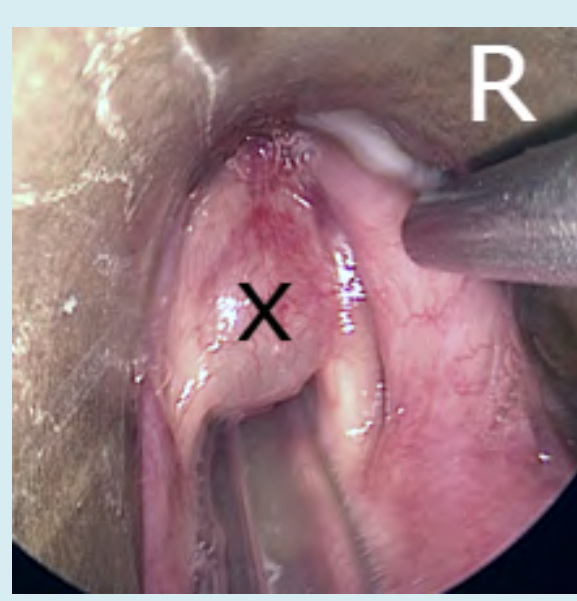

Figure 1: Intra - operative picture of the left vocal fold cystic lesion $(\mathrm{X})$.

Histopathological examination of the $15 \mathrm{~mm}$ left vocal fold lesion showed stroma expanded with benign looking spindle cells suggestive of a neurofibroma. There was no evidence of dysplasia or malignancy. Markers were performed later for panCytokeratin, S100 and Desmin were negative. Ki67 proliferation rate was very low. The final histopathology report suggested the diagnosis of neurofibroma. The tonsillar lesion proved to be a benign apocrine cyst.

The patient was followed up in the clinic 4 weeks after her surgery. She reported that her voice was back to normal and the Fibro-optic Nasal Endoscope (FNE) examination revealed a properly healed vocal fold with no phonatory gap (Figure 2). Considering her diagnosis, a detailed history and physical examination was carried out to exclude the possibility of neurofibromatosis. She did not have any signs or symptoms suggestive of neurofibromatosis and no further investigations were performed. The case was also discussed in the head and neck multi-disciplinary team (MDT) meeting, in which a long-term follow up was recommended due to the possibility of recurrence. The patient was followed up at 6 months and 1 year after the surgery with no symptoms or evidence of recurrence seen on examination.

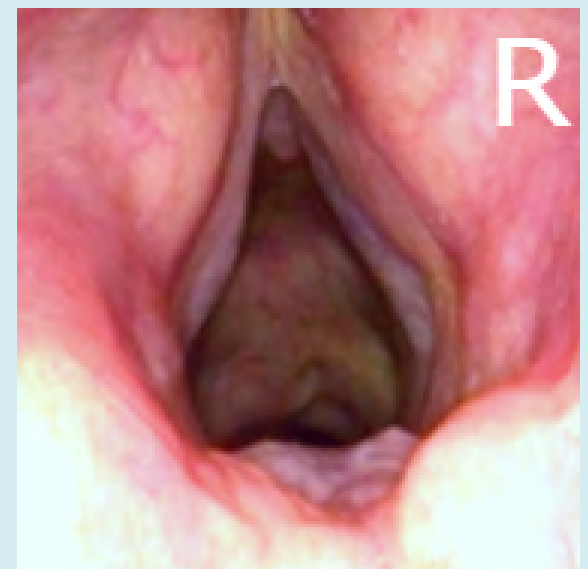

Figure 2: 1-year post operative picture of the larynx with no videnee of disease reeurrence.

\section{Discussion}

Laryngeal neurofibromas are benign tumours that originate from the peripheral nerve sheaths of the laryngeal nerves. Although $25 \%$ to $35 \%$ of the neurofibromas are found in the head and neck area[4], they make up only $0.1 \%$ of all the benign laryngeal tumours [4]. Despite the fact that most of the laryngeal neurofibromas are part of neurofibromatosis disease (Von Recklinghausen's disease), it can present as a solitary lesion. The most commonly involved part of the larynx is the supraglottis which includes the aryepiglottic fold and the arytenoid, as it has very rich nerve supply from the superior laryngeal nerve [6-9]. This is followed by the subglottic area which is supplied by the branches of the recurrent laryngeal nerve [9]. The only reported case from the glottis postulates that it can arise from encapsulated neural structures which are embedded in the connective tissue of the vocalis muscle [2].

Literature review showed that more than 100 cases with laryngeal neurofibroma were reported, [2-13] 62 of them were in the paediatric age group [7]. Only 15 reported cases had isolated laryngeal neurofibroma lesions without neurofibromatosis [8], and only two of them had isolated true vocal cord neurofibroma [2]. Our patient is the third reported case in the literature with isolated laryngeal neurofibroma of the true vocal fold without neurofibromatosis disease.

The symptoms of laryngeal neurofibromatosis depend on the age of the patient and the location of the lesion. Dysphonia is the most common presenting symptom in adult age group [10], while in children, stridor is the most common one [7]. Other symptoms can include dyspnoea, dysphagia, cough, chocking, globus sensation, recurrent throat clearing 


\section{Medical Journal of Clinical Trials \& Case Studies}

and sleep apnoea. Some patients can be asymptomatic, and the lesion can be discovered incidentally [8].

Definitive diagnosis should be made by direct endoscopic examination with excisional biopsy and histopathological examination, but pre-operative imaging can be useful in cases with large lesion or extensive disease to confirm the extension, vascularity of the lesion and planning for the appropriate surgical approach. On CT scan, the neurofibroma appears with a peripheral hypodensity and central enhancement [10]. The appearance on the MRI scan depends on the tissue consistency (fibrous vs. neural) $[9,11]$. Generally, MRI of the neurofibroma will show isointense or hypointense lesion on T1 weighted images and hyperintense on the $\mathrm{T} 2$ weighted images [7].

There are no established guidelines for treating cases of laryngeal neurofibroma, but complete surgical excision is the treatment of choice. For localised small lesions, like our patient, transoral endoscopic approach with cold dissection or $\mathrm{CO} 2$ laser is enough for complete excision and preservation of the laryngeal function. For more extensive disease, open approach with a laryngo-fissure might be needed $[7,12]$.

Significant complications that can develop after the surgery include vocal fold paralysis, intra-operative bleeding, scarring, vocal cord paresis or paralysis, permanent voice change and laryngeal stenosis $[10,12]$.

Although it is rare after complete surgical excision, but delayed recurrence of the disease has been reported previously [13]. No malignant transformation was mentioned in the literature for solitary laryngeal neurofibroma cases, but $5 \%$ of the neurofibromas developed malignant sarcoma especially when associated with neurofibromatosis disease [12], with 5 years survival rate of $20 \%-50 \%$ [16]. Therefore, long term follow up with direct visualisation by Fibre-optic Nasal Endoscope (FNE) examination is essential in these cases.

\section{Conclusion}

Despite the rarity of the disease, neurofibroma of the larynx can present as a solitary lesion in any age group, without the association with neurofibromatosis disease. Complete surgical excision by endolaryngeal endoscopic approach is the treatment of choice for small localised lesions. With long term follow up is recommended due to the possibility of recurrence.

\section{References}

1. Jones SR, Myers EN, Barnes L (1984) Benign neoplasms of the larynx. Otolaryngol Clin North Am 17(1): 151-178.
2. Liu J, Wong CF, Lim F, Kanagalingam J (2013) Glottic Neurofibroma in an Elderly Patient: A Case Report. J Voice 27(5): 644-646.

3. Sunny FA, Jishana J, Naik PP, Kurien M (2019) Shredded carrots in the larynx: A rare case of neurofibroma of vocal cord. J Curr Res Sci Med 5(1): 65-67.

4. Maisel RH, Ogura JH (1974) Neurofibromatosis with Laryngeal Involvement. Laryngoscope 84(1): 132-155.

5. Yoshida T, Kuratomi K, Mitsumasu T (1983) Benign Neoplasms of the Larynx. A 10-Year Review of 38 Patients. Auris Nasus Larynx 10: S61-71.

6. Chang-Lo M (1977) Laryngeal involvement in Von Recklinghausen's disease: a case report and review of the literature. Laryngoscope 87(3): 435-442.

7. Chinn SB, Collar RM, McHugh JB, Hogikyan ND, Thorne MC (2014) Pediatric laryngeal neurofibroma: case report and review of the literature. Int J Pediatr Otorhinolaryngol 78(1): 142-147.

8. Naunheim MR, Plotkin SR, Franco RA, Song PC (2016) Laryngeal Manifestations of Neurofibromatosis. Otolaryngol-Head Neck Surg 154(3): 494-497.

9. Nakahira M, Nakatani H, Sawada S, Matsumoto S (2001) Neurofibroma of the Larynx in Neurofibromatosis: Preoperative Computed Tomography and Magnetic Resonance Imaging. Arch Otolaryngol Neck Surg 127(3): 325-328.

10. Zhang L, Jiang J, Hu C, Yang H, Deng P, et al. (2018) Diagnosis and Management of Solitary Laryngeal Neurofibromas. Am J Med Sci 356(1): 79-83.

11. Rahbar R, Litrovnik BG, Vargas SO, Robson CD, Nuss RC, et al. (2004) The biology and management of laryngeal neurofibroma. Arch Otolaryngol Head Neck Surg 130(12): 1400-1406.

12. Willcox TO, Rosenberg SI, Handler SD (1993) Laryngeal involvement in neurofibromatosis. Ear Nose Throat J 72(12): 811-2, 815.

13. Koc C, Luxenberger W, Humer U, Friedrich G (1996) Bilateral ventricular neurofibroma of the larynx. J Laryngol Otol 110(4): 385-386.

14. Anagiotos A, Beutner D (2015) Laryngeal neurofibroma. Ear Nose Throat J 94(10-11): 436.

15. Supance JS, Quenelle DJ, Crissman J (1980) Endolaryngeal neurofibromas. Otolaryngol Head Neck Surg 88(1): 7478. 


\section{Medical Journal of Clinical Trials \& Case Studies}

16. White AK, Smith RJH, Bigler CR, Brooke WF, Schauer PR (1986) Head and Neck Manifestations of
Neurofibromatosis: The Laryngoscope 96(7): 732-736.

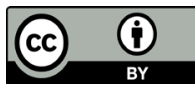

